\title{
Konfliktbeladene Abhängigkeiten: Wie der chinesische Aufstieg weltwirtschaftliche Kräftekonstellationen verändert
}

\author{
Tobias ten Brink
}

Zusammenfassung: Im Anschluss an politökonomische Ansätze wird die Volksrepublik China als eine Variante der nachholenden (staats-)kapitalistischen Entwicklung analysiert, die an einer wirtschaftlichen und politischen Aufwertung interessiert ist. Dabei verkörpert die inter- und transnationale Einbettung des chinesischen Kapitalismus in ein instabiles Weltsystem trotz zahlreicher ökonomischer Interdependenzen und des Bemühens der chinesischen Staatsführung um eine ,verantwortungsvolle“ Außenpolitik einen spannungsreichen Prozess.

Schlüsselwörter: China $\cdot$ Ostasien $\cdot$ Kapitalismus $\cdot$ Außenwirtschaftspolitik $\cdot$ Währungssystem

\section{Strained Dependencies. How the Rise of China Is Changing Global Economic Power Constellations}

\begin{abstract}
Referring to theories of political economy, this paper analyses the People's Republic of China as a variety of a (state-led) capitalist catch-up development process that is focused on economic and political upgrading. Despite numerous economic interdependencies as well as efforts by China's political leadership to act as a „responsible“ power, the international and transnational embedding of the new Chinese capitalism into an unstable world system is fraught with tension.
\end{abstract}

Keywords: China $\cdot$ East Asia $\cdot$ Capitalism $\cdot$ Foreign economic policy $\cdot$ Currency system

\section{Article by an MPIfG researcher}

Tobias ten Brink: Konfliktbeladene Abhängigkeiten: Wie der chinesische Aufstieg weltwirtschaftliche Kräftekonstellationen verändert. In: Zeitschrift für Außen- und Sicherheitspolitik 5(4), 625-643 (2012). VS Verlag für Sozialwissenschaften

The original publication is available at the publisher's web site: http://dx.doi.org/10.1007/s12399-012-0287-y

(C) VS Verlag für Sozialwissenschaften 2012

Dr. T. ten Brink $(\bowtie)$

Goethe-Universität Frankfurt am Main,

Robert-Mayer-Str. 5, 60054 Frankfurt am Main, Deutschland

E-Mail: tobias.ten.brink@em.uni-frankfurt.de 


\section{Einleitung}

In den letzten beiden Jahrzehnten ist die Volksrepublik (VR) China zur zweitgrößten Wirtschaft der Erde avanciert. Chinas Aufstieg steht dabei am Ende einer Reihe von vergleichsweise erfolgreichen nachholenden Ökonomien in Ostasien, die einen Trend zu einer Rezentrierung der Weltwirtschaft auslösten. Die zunehmende Bedeutung der chinesischen Volkswirtschaft hat eine Restrukturierung der Konkurrenzverhältnisse auf den internationalen Märkten zur Folge. Jüngst lässt sich ebenso eine zugunsten Chinas (und anderer Schwellenländer) geänderte Kräftekonstellation in den internationalen Institutionen feststellen, wie der im Zuge der globalen Finanz- und Wirtschaftskrise neu begründete G20-Gipfel und eine Stimmrechtsreform des Internationalen Währungsfonds (IWF) nahelegen.

Über die Folgen dieses epochalen Wandels sind sich Journalisten und Sozialwissenschaftler, besonders in der Disziplin der Internationalen Beziehungen (IB), uneinig. Grob lassen sich zwei Positionen idealtypisch kontrastieren: Während etliche Autoren vor einer chinesischen „Konfrontation“, „Gefahr“ und/oder „Herausforderung“ warnen, erwarten zahlreiche andere Autoren eine vorwiegend kooperative Integration Chinas in die Weltwirtschaft und -politik.

Die erste Position drückt oft ein tief sitzendes Misstrauen gegenüber einer herrschsüchtigen Kommunistischen Partei (KPCh) aus, die nur bedingt gewillt sei, ihre neu gewonnenen Machtkapazitäten ausschließlich friedvoll einzusetzen. Unter Verweis auf die guten Beziehungen Chinas zu „Schurkenstaaten“ wird auf die Dissonanz zwischen Rhetorik und Praxis der Staatsführung verwiesen. Ein sich entwickelnder „Beijing Consensus“, ein Entwicklungsmodell, das auf Innovation durch Mischeigentum, schwache private Eigentumsrechte, Staatsunternehmen und Staatsinterventionismus abzielt, bedrohe den westlich-liberalen „Washington Consensus“ und führe zu Verwerfungen im globalen Institutionengefüge, so lautet ein weiteres bekannt gewordenes Argumentationsmuster (Bremmer 2010; Halper 2010). In den Politikwissenschaften und der Disziplin der IB wird das Ende der amerikanischen Hegemonie beziehungsweise ein konfliktiver Übergang in ein chinesisches Jahrhundert antizipiert und/oder auf Unsicherheiten verwiesen, die historisch immer wieder zwischen aufstrebenden und dominierenden Mächten auftraten und in Großkonflikte mündeten (Arrighi 2008; Layne 2009). Offensive Neorealisten wie John J. Mearsheimer spitzen dieses Argument noch zu: Sollte das chinesische Wirtschaftswachstum anhalten und die Staatsführung die Streitkräfte modernisieren, müsse von unvermeidlichen Konflikten bis hin zum Krieg mit den USA ausgegangen werden (Mearsheimer 2010).

Demgegenüber stehen Positionen, die in unterschiedlicher Weise eine weitaus harmonischere Lage diagnostizieren. Eine Integration Chinas in die Weltwirtschaft und partnerschaftlich-kooperative Beziehungen mit dem Westen erscheinen solchen Autoren wahrscheinlicher, die auf eine Exportabhängigkeit Chinas sowie eine pragmatische Orientierung auf wirtschaftliches Wachstum verweisen, welche nationale Sicherheitsinteressen und geopolitische Machtstrategien in den Hintergrund treten lassen: Sowohl transnationale Unternehmen als auch die chinesische Staatsführung würden von einer Integration Chinas in das westlich dominierte globale Institutionensystem profitieren (Ikenberry 2008; Jiang 2008; Wang und Rosenau 2009). Die Befolgung internationaler 
Normen und Regeln habe für das Reich der Mitte fast ausschließlich Vorteile mit sich gebracht. China strebe aus diesem Grund keine Konfrontation mit anderen Mächten und ebenso wenig eine Abkopplung vom internationalen System an. Die chinesische Außenpolitik setze vorwiegend auf die Instrumente der soft power, um Interessen durchzusetzen (Kurlantzick 2008). Ein Machtungleichgewicht in Ostasien könne durch eine regionale Integration nach dem Vorbild der EU unterbunden werden, moderiert durch eine durchdachte Balancing-Politik der USA.

Besitzt der Begriff von der chinesischen „Herausforderung“ also lediglich demagogische Züge? Oder stellen umgekehrt die optimistischen Integrationserwartungen regelrecht idealistische Perspektiven dar? Tatsächlich hat der Aufstieg Chinas eine komplexe Gemengelage mit kooperativen und konfliktiven Aspekten geschaffen, deren gegenwärtige Beschaffenheit nun in folgender Weise untersucht wird: In einem ersten Teil frage ich, um welches Modell gesellschaftlicher Entwicklung es sich in der Volksrepublik eigentlich handelt. Im Anschluss an neuere Erkenntnisse der Chinaforschung und politökonomischer Ansätze wird die VR China als Variante der nachholenden (staats-) kapitalistischen Entwicklung analysiert (Kap.2). Enge Verknüpfungen staatlicher und privater Akteure kennzeichnen dieses Modell ebenso wie eine strenge Wachstums- und Wettbewerbsorientierung sowie eine offensive nationale Ausrichtung in der Außen- und Außenwirtschaftspolitik.

Daraufhin wird die inter- und transnationale Einbettung des neuen chinesischen Kapitalismus in ein instabiles Weltsystem untersucht (Kap.3). Über den Fortbestand des pluralen Staatensystems und verschiedener, nicht immer komplementär verbundener nationaler Standort- und weiterer Außenpolitiken hinaus ist dieses durch Verschiebungen der internationalen Kräftekonstellationen charakterisiert. Die Fragilität des Weltsystems unter Führung der Vereinigten Staaten befördert, so eine Hypothese, spannungsbehaftete Handlungen Chinas, auch wenn das Land sich um eine ,verantwortungsvolle“ Großmachtpolitik bemüht. Auf dieser Grundlage wird nach der empirischen Verifizierbarkeit der Hypothese eines konfliktbeladenen Aufstiegs der Volksrepublik gefragt und dem Verhältnis zu den USA Aufmerksamkeit geschenkt. Zwar bleibt die chinesische Volkswirtschaft abhängig von den ,alten“ Zentren des globalen Kapitalismus bzw. von transnationalen Konzernen und westlichen Exportmärkten. Daraus folgt jedoch nicht umstandslos eine reibungslose Zusammenarbeit. Im Gegenteil weisen aktuelle Währungsdispute im Zuge des globalen Einbruchs 2008/2009 auf politisch vermittelte Konflikte relevanter Wirtschaftsstandorte hin. Optimistische Perspektiven einer konfliktarmen Integration Chinas müssen daher relativiert werden - wie zusätzlich die Führungsrolle des Landes im ostasiatischen Regionalisierungsprozess illustriert -, ohne voreilige Schlussfolgerungen hinsichtlich militärischer Zusammenstöße und eines Übergangs zu einer neuen globalen chinesischen Hegemonie zu übernehmen, die in Ostasien ihren Ausgangspunkt nimmt. Der neue chinesische Machtpol ${ }^{1}$ bleibt in regionale und globale Kooperationsgeflechte

1 Dass Machtpole auch in einer entstehenden „Weltgesellschaft“ in hohem Maße mit Staaten gleichgesetzt werden, ist eine Folge der fortwährenden Bedeutung des internationalen Staatensystems. Die geschichtliche Entwicklung mündete nicht in die Organisation eines mit den Kapazitäten von starken Einzelstaaten ausgestatteten supranationalen Staates. Auch in der fortgeschrittenen Phase der Globalisierung lassen sich zwei relativ unabhängig voneinander 
eingebunden, auch wenn dieser etablierte Kräftekonstellationen in den internationalen Institutionen, etwa über seine Blockademacht, infrage stellt. Ein hegemonialer Übergang und manifeste geopolitische Konflikte mit den USA sind gegenwärtig - auch in Ostasien - wenig wahrscheinlich. ${ }^{2}$

Theoretisch knüpfen die folgenden Überlegungen an Einsichten der Chinaforschung, der Vergleichenden Politischen Ökonomie (VPÖ), der Internationalen Politischen Ökonomie (IPÖ) sowie an eigene Vorarbeiten an (ten Brink 2008, 2011, 2012a, b). Zahlreiche Studien, die den Umstrukturierungen Ostasiens, der Weltwirtschaft und regionalen bzw. internationalen Institutionen, Regulierungs- und Hegemonialstrukturen Rechnung tragen, dienen als weitere Bezugspunkte (vgl. Beeson 2009; Breslin 2010; Dittmer und Yu 2010; Katzenstein und Shiraishi 2006). Abgezielt wird darauf, die Vorteile einer politökonomischen Betrachtungsweise bei der Beantwortung einer Frage herauszustellen, die üblicherweise in einer „IB-Perspektive“, d.h. fokussiert auf zwischenstaatliche Beziehungen, behandelt wird.

\section{Nachholende (staats-)kapitalistische Entwicklung}

Als die chinesische Machtelite im Jahr 1978 unter Führung des Reformflügels um Deng Xiaoping begann, das nach der Kulturrevolution sich in einer schweren gesellschaftlichen Krise befindende Land in einem Trial-and-error-Verfahren zu restrukturieren, konnte niemand ahnen, was das Ergebnis dieser Wende sein würde. Resultat mehrerer tief greifender Veränderungen ist eine bislang einmalige Transformation einer Kommandowirtschaft in ein stärker über den Markt gesteuertes Entwicklungsmodell.

Ein Bezug auf die offizielle Losung der Kommunistischen Partei, die das Land zur „sozialistischen Marktwirtschaft“ erklärt hat, greift zu kurz. Typische Kennzeichen des Kapitalismus wie der Zwang zur Akkumulation des Kapitals, eine end- und maßlose Wachstumsorientierung, Wettbewerbsprinzipien, zweckrationale Nützlichkeitserwägungen sowie ausgeprägte soziale Gegensätze haben die Ökonomie zu einem neuen Zentrum des globalen Kapitalismus gemacht. Aus diesem Grund ist es hilfreich, zur Untersuchung der nachholenden Entwicklung Chinas auf Erkenntnisse der VPÖ und IPÖ zurückzugreifen. Im Unterschied zu markt- und unternehmenszentrierten Kapitalismustheorien orientiere ich mich dabei an einem erweiterten Forschungsrahmen zur Analyse kapitalistischer Entwicklungen, der staatliches Handeln und internationale Machtbeziehungen integriert. Zudem knüpfe ich an das Konzept eines variegated capitalist world system an, um Gemeinsamkeiten der globalkapitalistischen Entwicklung und sozioökonomische

existierende und nicht aufeinander zu reduzierende Muster der sozioökonomischen Unternehmens- sowie der (geo-)politischen Staatenkonkurrenz nachweisen (vgl. ten Brink 2008; ten Brink 2012a; sowie die in unterschiedlicher theoretischer Perspektive vorgetragenen Aufsätze zur fortwährenden Bedeutung des Staatensystems: ten Brink 2011).

$2 \mathrm{Zu}$ weiteren, auch explizit geopolitischen Implikationen des chinesischen Machtzuwachses, die zur Infragestellung abgesteckter Interessensphären ,alter“ Großmächte führten und bereits manifestere Konflikte als in einigen der hier erläuterten Beispielen zur Folge hatten, besonders in Afrika, vgl. Alden (2010) sowie Dittmer und Yu (2010). Die Spannungsverhältnisse Chinas mit anderen Staaten (etwa mit Indien) bleiben hier aus Platzgründen unberücksichtigt. 
Interdependenzen zwischen verschiedenen Kapitalismen zu erfassen, die jedoch immer wieder auch Konflikte hervorrufen (vgl. Jessop 2009; Streeck 2010).

Die politische Ökonomie Chinas wird des Weiteren als wettbewerbsgetriebene Variante eines heterogenen, staatlich gelenkten Kapitalismus betrachtet (vgl. ten Brink 2012b). Ein wettbewerbsgetriebener, unternehmerischer Geist ist mit einem umfassenden Staatsinterventionismus verbunden. Die Führung des Landes setzt dabei zugleich auf eine wirtschaftliche und eine geopolitische Aufwertung. Enge Verflechtungen zwischen Staatsapparaten und Unternehmen kennzeichnen die chinesische Gesellschaft - ein Erbe der bürokratischen Kommandowirtschaft, der herrschenden KPCh und der Rolle eines industriellen Spätentwicklers (McNally 2007; Naughton 2007). Gleichwohl ist der chinesische Kapitalismus tief eingebettet in weltwirtschaftliche Prozesse und abhängig gegenüber den Märkten großer OECD-Volkswirtschaften sowie von ausländischen Direktinvestitionen.

Um Merkmale der nachholenden kapitalistischen Entwicklung Chinas detaillierter kennzeichnen zu können, werden nun 1) wichtige Kennzeichen der Ökonomie, 2) das Wechselverhältnis zwischen Staat und Wirtschaft und 3) das Finanzsystem kursorisch beschrieben. ${ }^{3}$

Kennzeichen der Ökonomie: Die systemische Notwendigkeit der erweiterten Akkumulation und Innovation mit dem Ziel der Profitmaximierung setzt sich in der Volksrepublik vor dem Hintergrund einer Verschiebung der wirtschaftlichen Verfügungs- und Kontrollkapazitäten von staatlichen Behörden auf das Management zahlloser Unternehmen durch. Eine weder „frei“ noch klassisch „kompetitive“ gemischte Wirtschaft regelt diese Beziehungen. Während auf der zentralstaatlichen Ebene eine erneuerte Form des (profitorientierten) staatseigenen Unternehmens dominiert, konnten besonders auf subnationalen Ebenen private Unternehmen und privat-öffentliche Wirtschaftsregime an Bedeutung gewinnen. Die nahezu symbiotischen Beziehungen zwischen Partei, Staat und Unternehmen lösten sich dabei nicht auf. Zugleich ist die chinesische Volkswirtschaft als „Werkbank der Welt" in den letzten Jahrzehnten weitgehend in den Weltmarkt und in die Netzwerke transnationaler Produktionsverbünde integriert worden. ${ }^{4}$

3 Die für kapitalistische Systeme ebenfalls kennzeichnenden hierarchischen Arbeitssysteme sowie soziale Destabilisierungsdynamiken bleiben an dieser Stelle unterbelichtet. Soziale Stratifizierungen und insbesondere die vertikale „Klassenpolarisierung zwischen Vermögensbesitzern und Vermögenslosen“ (Deutschmann 2009, S. 38) bilden eine Basis fortwährender innergesellschaftlicher Auseinandersetzungen, die Legitimationskrisen auslösen können. Relevant für die Frage nach den Effekten des internationalen Aufstiegs Chinas ist in diesem Zusammenhang der bereits in anderen Gesellschaften beobachtete Sachverhalt, dass interne gesellschaftliche Konflikte und Legitimationsdefizite vielfach von Machteliten dadurch zu lösen versucht werden, dass ein externes, feindliches „Anderes“ konstruiert wird. Ein innenpolitischer Konsens wird über eine Abgrenzung nach außen bzw. nach außen zielende Bedrohungskonstruktionen herzustellen versucht.

4 Nationale Datensätze spiegeln nur unzureichend die transnationale Organisation der chinesischen Wirtschaft wider. Viele der in China erzeugten Exportprodukte sind nur in dem Sinne „chinesisch“, dass sie hier montiert werden. 
Aus diesen Dynamiken resultieren eine Reihe von Wettbewerbsstrategien, unter anderem der Versuch chinesischer Konzerne, in westlich dominierten Wertschöpfungsketten Einfluss zu gewinnen, eine Exportoffensive und, seit einigen Jahren, die internationale Verlagerung von Kapital sowie, auf einer Analyseebene, die staatliche Wirtschaftspolitiken berücksichtigt, die politische Unterstützung von internationalen Unternehmensaktivitäten, der Zugang zu und die Sicherung von Rohstoffen sowie die Lenkung von Investitionen.

Partei-Staat und Wirtschaft: Die Bedeutung der Doppelstruktur von Partei und Staatsapparaten sowie unterschiedliche Grade und Formen der Staatsintervention begründen ein distinktes politisches System auf dem chinesischen Festland: den „Partei-Staat“. Die Parteistaatsideologie und -praxis hat sich in den letzten Jahrzehnten erneuert. Der Begriff des Sozialismus steht nunmehr vor allem anderen für eine gelingende wirtschaftliche Modernisierung und pragmatische, experimentierfreudige Handlungsorientierungen. Partei- bzw. Staatsapparate orientieren sich an makroökonomischen Erfolgsparametern. Die Staatsintervention sowie das Staatseigentum in der Volksrepublik stellen keine Negation kapitalistischer Verhältnisse dar.

„Wirtschaft“ und „Politik“ bilden in China ein interdependentes Geflecht. Empirischen Untersuchungen zufolge führten die Transformationsprozesse zu engen Interessenkoalitionen zwischen ökonomischen und politischen Eliten. Staatliches Handeln spielt bei der Gestaltung wirtschaftlicher Vorgänge eine konstitutive Rolle. Umgekehrt befinden sich die Staatsapparate in einer Abhängigkeit von einer gelingenden Akkumulation, weshalb (beinahe) alles unterbunden wird, was die ökonomische Entwicklung von Unternehmen gefährden könnte. Besonders ist das für Offizielle der unteren Gebietskörperschaften relevant, deren Karrierepfade direkt an hohe Wachstumsraten geknüpft sind. Doch auch die politische Machtelite insgesamt ist an einer gedeihlichen volkswirtschaftlichen Entwicklung - der Legitimitätsressource des Regimes schlechthin - und einer durchgreifenden nationalen Entwicklung in höchstem Maße interessiert, was die Vermutung nahelegt, dass außen(-wirtschafts-)politische Handlungen von diesen Motiven nicht grundlegend abweichen dürften. ${ }^{5}$

Das chinesische Finanz- und Geldsystem: Ein weiterer Kernbestandteil des chinesischen Wirtschaftssystems, der internationalen wirtschaftlichen Austausch ermöglicht, ihn aber zugleich konfliktiv strukturiert, wie in jüngster Zeit Währungsdispute zwischen den größten Volkswirtschaften der Erde signalisieren, ist das Finanz- und Geldsystem (Naughton 2007, S. 449-481). Es ist durch eine strategische Rolle des Staates charakterisiert. Im Mittelpunkt befindet sich ein Bankensystem, mit einer mächtigen Zentralbank, die die Geldpolitik bestimmt und den Wechselkurs kontrolliert. Neben den größten staatlichen, mittlerweile börsennotierten Geschäftsbanken und weiteren, zentralstaatlichen policy banks behaupten sich zunehmend weitere kommerzielle, meist börsennotierte Banken. Vor allem aus der Asienkrise 1997/1998 hat die chinesische Regierung hinsichtlich

5 Zugleich erklärt die hohe Abhängigkeit vom Export und gegenüber ausländischen Investitionen die Förderung ausländischer Unternehmen. Die Provinz- oder lokalen Stadtregierungen haben derart enge Verbindungen mit ausländischen Unternehmen geknüpft. 
der Geld- und Währungspolitik Lehren gezogen. Die staatliche Kontrolle des Kapitalverkehrs und der Wechselkurse soll aufrechterhalten bleiben.

Insgesamt verkörpert der in den Weltmarkt eingebundene, staatlich gelenkte Kapitalismus in China bis dato ein überaus dynamisches Wirtschaftsmodell. Zugleich ist sich die regierende, technokratische Machtelite der weltwirtschaftlichen und -politischen Abhängigkeiten bewusst, die die weitere chinesische Entwicklungsdynamik blockieren können.

\subsection{Chinas Aufstieg: Außenpolitische Flankierungen}

Die weitgehende Abhängigkeit des chinesischen Wirtschaftsraums von weltwirtschaftlichen Entwicklungen übersetzt sich in folgendes außenpolitisches Selbstverständnis: Die chinesische Staatsführung artikuliert sich als eine aufstrebende, jedoch „verantwortungsvolle" Großmacht, die sich den Zielen der ökonomischen Stabilität, des Wachstums und des globalen Friedens verpflichtet fühlt. Das innenpolitische Credo einer „harmonischen“ Gesellschaft bestimmt dabei die außenpolitischen Stellungnahmen. Da dem chinesischen Aufstieg international beständig mit Skepsis begegnet wird, stellt die Vorstellung einer „harmonischen Welt“ jedoch nicht zuletzt auch den Versuch dar, Ressentiments zu entkräften und einer antichinesischen Eindämmungspolitik wirksam zu begegnen. Daneben verweisen die Pekinger Außenpolitikstrategen auf die negativen Folgen aggressiver USamerikanischer Außenpolitik, setzen auf das UN-System als Zentrum der global governance, das Prinzip der Nichteinmischung in die inneren Angelegenheiten anderer Staaten und betonen die Diversität der Zivilisationen, was Spielräume für die nationale Auslegung internationaler Normen eröffnet (Jiang 2008).

Zugleich jedoch ist sich die auf engen Allianzen mit Unternehmen basierende Staatsführung der neuen Machtkapazitäten bewusst, die das historische Wachstum der Wirtschaft mit sich brachte. Um der aufholenden Entwicklung zum weiteren Erfolg zu verhelfen, bedienen sich die Staatseliten, vor dem Hintergrund der Kräfteverschiebungen im Weltsystem und der Existenz eines Standortwettbewerbs, folgender außenpolitischer Maßnahmen:

- Politische Institutionen leisten mit außenwirtschaftspolitischen Mitteln Hilfestellungen bei der globalen Suche nach rentablen Investitionsorten und aussichtsreichen Märkten. Als Beispiele hierfür gelten die Unterstützung bei der Durchführung von Auslandsinvestitionen oder diplomatisch vermittelte Wirtschaftsbeziehungen. Der chinesische Staatsrat und die Ministerien, z. B. das Handelsministerium MOFCOM, die Zentralbank oder die Nationale Entwicklungs- und Reformkommission NDRC, verfügen ferner über weitere Regulierungsinstrumente zur Stimulierung der Volkswirtschaft, insbesondere handelspolitische (wie tarifäre und nicht-tarifäre Handelshemmnisse, Zölle) und währungspolitische (Beeinflussung des Wechselkurses, Geldpolitik). Die Regierung fördert in der Regel ausländische Investitionen in das eigene Territorium, zugleich kontrolliert sie jedoch auch nach dem WTO-Beitritt 2001 die Marktzugangskriterien und bestimmte Schlüsselbereiche sind nur eingeschränkt zugänglich (Breslin 2010). ${ }^{6}$

6 Entgegen der verbreiteten Vorstellung eines monolithischen Politikfindungsprozesses in China führt die Fragmentierung der chinesischen Gesellschaft in ein komplexes Mehrebenensystem 
- Darüber hinaus wird mit der Sicherheitspolitik der Versuch eines Managements der internationalen Beziehungen geleistet, der eine gelingende Kapitalakkumulation zumindest indirekt zu unterstützen beabsichtigt. Das reicht von der gezielten Förderung der Sicherung der Rohstoffversorgung über weiche (geo-)politische Maßnahmen wie ökonomische Anreize bis hin zu harten geopolitischen Handlungen wie der Sicherung von Handels- und Seewegen. ${ }^{7}$ Die militärischen Fähigkeiten des Staates verkörpern insofern „diskrete“ Hintergrundinformationen für sein internationales Durchsetzungsvermögen (Zhang 2010, S. 49-57).

Diese Instrumente beeinflussen die Position Chinas im internationalen System. Ähnlich wie andere aufholende Staaten und Großmächte zielt die Volksrepublik auf die Agglomeration umfassender ,nationaler Stärke““ ${ }^{8}$ Um dieses Ziel zu erreichen, tritt die VR China für eine multilaterale Entscheidungsfindung ein. Betont werden „weiche“ Machtinstrumentarien und die Kraft einer abgestimmten global governance. Genutzt werden jedoch gleichwohl eine gewachsene Blockademacht in den internationalen Institutionen und „harte“ Machtinstrumente, wie an der Modernisierung der Armee und der Schaffung einer starken Seemacht im Südchinesischen Meer und dem Indischen Ozean abzulesen ist, um als legitim erachteten Sicherheitsinteressen Nachdruck zu verleihen. Eine „gebührende“ Stellung auf der internationalen Bühne beruht allem Anschein nach auch auf jenen harten, nicht immer harmonisierenden Maßnahmen, die in Peking lediglich rhetorisch zunehmend als anachronistisch betrachtet werden (vgl. Wang und Rosenau 2009, S. 36-38).

Freilich steht dem chinesischen Machtapparat jedoch zugleich ein noch weitaus stärkerer Machtkomplex zuweilen misstrauisch gegenüber: Begleitet von diplomatischen Beschwichtigungsgesten führt die Obama-Administration, anknüpfend an liberal-realistische Traditionslinien, Sicherheitsmaßnahmen fort, um China in eine Art Konzert der Mächte einzuordnen - dirigiert durch die USA, mit dem unausgesprochenen Ziel einer Unterordnung oder Domestizierung der Volksrepublik. Ende 2011 wurde in diesem Zusammenhang eine Verstärkung der Truppenpräsenz in der asiatisch-pazifischen Region, auch im wichtige maritime Verkehrswege umfassenden Südchinesischen Meer, beschlossen, was in China als Provokation bewertet wird. Alles in allem erzeugt diese, hier holzschnittartig geschilderte Konstellation wirtschaftliche und außenpolitische

auf außenpolitischer Ebene dazu, dass immer wieder Kompetenzkonflikte nicht nur zwischen Außen- und Handelsministerium oder zwischen Militärs und ziviler Obrigkeit, sondern auch zwischen den zentralstaatlichen und regionalen Regierungen aufbrechen. Die außenpolitische Willensbildung ist demnach erheblich komplexer (und führt mitunter zu widersprüchlichen Signalen, policies oder auch bürokratischer Immobilität), als an dieser Stelle nachgezeichnet werden kann (vgl. Schmidt 2012).

7 Generell lassen sich geopolitische „Dienstleistungen“ und Strategien selbstverständlich nicht hinreichend ökonomisch ableiten, sondern sind immer auch Ausdruck der Interessen einzelstaatlicher Instanzen, die damit etwa auf die Aufrechterhaltung ihrer Souveränität und damit ihrer Machtbasis zielen.

8 In innerchinesischen Debatten in der Disziplin der IB lässt sich ein großes Interesse an great power politics nachweisen (Huang 2007). Beispielhaft ist der Artikel des einflussreichen Parteiintellektuellen Zheng (2005), der die These eines friedlichen Aufstiegs zur Großmacht vertritt. 
Spannungen, obgleich ökonomische Verflechtungen, Interdependenzen sowie temporäre Win-win-Situationen bestehen und ein behutsamer Machtgebrauch überwiegt.

\section{Die Einbettung des chinesischen Kapitalismus in ein fragiles Weltsystem}

Die chinesische Volkswirtschaft konnte in den letzten Jahrzehnten von günstigen weltwirtschaftlichen Rahmenbedingungen profitieren: von einer Verlagerung der Waren- und Wertschöpfungsketten in Richtung Ostasien etwa und von den in transnationale Produktionsverbünde eingebetteten Netzwerken der „Überseechinesen“, die seit den 1980er Jahren mit ihren Investitionen vor allem via Hongkong gewissermaßen Brücken auf das chinesische Festland bauten. Ab den 1990er Jahren, in denen sich das an ostasiatischen Vorbildern orientierte Exportregime Chinas etablierte, zehrte das Wirtschaftswachstum zudem von einer niedrigeren Investitionsquote in den stärksten OECD-Ländern. Eine in den klassischen Produktionszentren als Anlagenotstand deklarierte Überakkumulation von Kapital begünstigte die Kapitalverlagerung. Im Gefolge der Asienkrise 1997/1998 und später der Dotcom-Krise im Jahr 2001 orientierten sich die transnationalen Produktionsverbünde (vor allem die Elektronikindustrie) vorwiegend in Richtung des chinesischen Festlandes (Hung 2008).

Diese Restrukturierung der Kapitalverwertung unterstützte Chinas nachholende Entwicklung und verlieh dem bevölkerungsreichsten Land der Erde damit den Status eines überaus relevanten strategischen Standorts der einstigen Peripherie. Ähnlich wie in früheren Entwicklungsphasen des Kapitalismus konnte derart eine aufholende Wirtschaftsmacht von den Entwicklungsvorsprüngen der dominanten Ökonomien in der Entfaltung der Produktivkräfte profitieren: durch den Transfer von technischem und organisatorischem Know-how und, vor dem Hintergrund der Verlangsamung des Wachstums in den alten Zentren, vermittelt durch ausländische Direktinvestitionen.

Bis in das Jahr 2008 etablierte sich hierbei jedoch keineswegs eine hauptsächlich konfliktive Sachlage. Eher konnten die großen westlichen Ökonomien, besonders die Vereinigten Staaten und China ihr Wachstum auf wechselseitig voneinander abhängigen Wegen erzielen: Große Mengen an liquiden Mitteln auch und gerade aus den USA stellten die Versorgung mit Geldanlagen sicher und förderten den Investitionsboom in China. Zugleich fungierten die USA als ein Endabnehmer der innerhalb Chinas produzierten Exportwaren.

Doch nicht zuletzt der globale Einbruch 2008/2009 ließ Zweifel daran aufkommen, dass diese Win-win-Konstellation - ein geflügeltes Wort im heutigen China - auf Dauer gestellt werden könne (Schmalz 2011).

\subsection{Von der „Werkbank der Welt“ zum ernst zu nehmenden Konkurrenten}

Vor dem Hintergrund außen(-wirtschafts-)politischer Flankierungen chinesischer Interessen und der Fragilität des von den USA geführten Weltsystems lassen sich nun einige Folgen des chinesischen Aufstiegs genauer darlegen. Bezug nehmend auf die eingangs erörterten Kernbestandteile des neuen chinesischen Kapitalismus - eine tiefe weltwirtschaftliche Einbindung, zugleich jedoch eine Staatszentrierung, auch im Finanzsystem 
- werden im Folgenden, erstens, die auf Abhängigkeiten und kooperativen Aspekten beruhenden, doch gleichwohl spannungsreichen Effekte der Going-out-Strategie des chinesischen Kapitalismus herausgestellt. Dabei verkörpern die Währungsdispute mit den USA und der Streit um die Rolle des US-Dollars als Weltleitwährung ein Beispiel für die komplexe Vermittlung von ökonomischen Konkurrenzverhältnissen und politischen Kontrollstrategien. An der Rolle Chinas in den internationalen Organisationen und der ostasiatischen Regionalisierung demonstriere ich zweitens, dass die Außenpolitik Chinas bestimmte, nicht intendierte Nebenfolgen zeitigt, die Konflikte fördern.

Der globale Einbruch 2008/2009, der Kriseneffekte in den auf die US-amerikanischen bzw. westlichen Verbrauchermärkte orientierten chinesischen Branchen nach sich zog, hat die wechselseitige Abhängigkeit der beiden größten Volkswirtschaften der Erde eher noch verstärkt. Da der chinesische Binnenmarkt wächst und dort weiter ertragseffizient produziert werden kann, sehen westliche Unternehmen die chinesische Volkswirtschaft als Rettungsanker - auch wenn China nun einen noch größeren Teil des weltweiten Sozialproduktes auf sich vereint und über Geldanlagen verfügt, die in westlichen Medien regelmäßig als bedrohliche Machtkonzentration bewertet werden. Umgekehrt bleibt der chinesische „Exportismus“ abhängig von den US-amerikanischen bzw. westlichen Märkten.

Die wechselseitigen Abhängigkeiten bringen allerdings eine Machtverschiebung mit sich: Zwar sind die originär chinesischen Konzerne gegenwärtig noch meist als ausführende Objekte in die transnationalen Produktionsverbünde eingebunden und auch den Großteil der Profite erzielen die multinationalen Konzerne (Hürtgen et al. 2009). Doch die Unternehmen der entwickelten Volkswirtschaften schaffen sich auf diese Weise zugleich neue Konkurrenten in dem Maße, wie sie selbst versuchen, vom Wachstum auf dem chinesischen Festland zu profitieren (Zhu und Kotz 2011, S. 25-26).

Im Prozess der nachholenden kapitalistischen Entwicklung versuchen chinesische Konzerne sowie die eng mit diesen Unternehmen verbundene politische Machtelite in vielfältiger Weise die einstige, untergeordnete Bedeutung als verlängerte „Werkbank der Welt“ zu reduzieren: indem etwa Spill-over-Effekte genutzt werden, die sich positiv auf den technischen Fortschritt einheimischer Konzerne auswirken. Ähnlich wie deutsche Unternehmen im letzten Drittel des 19. Jahrhunderts englische Innovationen illegal kopierten, nutzen chinesische Konzerne und lokale Behörden beispielsweise Joint Ventures dazu, um den Technologietransfer zu beschleunigen. Ferner wurde und wird die chinesische Industrie durch den regen Kontakt mit ausländischen Großkonzernen an Weltmarktstandards angepasst, was wiederum einen Druck sowohl auf die Disziplinierung der Arbeitskräfte als auch auf die Entwicklung eines avancierten Ausbildungs- und Forschungssektors ausübt. Es bestehen umfangreiche Versuche, unternehmerische Schöpferkraft und staatliche Machtkapazitäten zu vereinen, um selbst zum Innovationsmotor zu werden.

In den letzten Jahren kam es daraufhin zu einer signifikanten Zunahme weltweit wettbewerbsfähiger staatlicher, halbstaatlicher und privater Firmen aus China (Yeung und Liu 2008). Im Gegensatz zum global rückläufigen Trend stiegen die chinesischen Auslandsdirektinvestitionen (ADI) nach 2008 erheblich an. Zwar fallen die internationalen Direktinvestitionen im Vergleich etwa zu den deutschen ADI noch geringer aus, doch in vielen Fällen verläuft diese Going-out-Strategie erfolgreich. Folgende Wege zur Ausweitung des internationalen Radius chinesischer Unternehmen lassen sich unterscheiden: 
- „Fokussierte“Exportunternehmen versuchen mit einem Produkt bzw. in einem Marktsegment, Weltmarktanteile zu erwerben. Die Firma Galanz steht als mittlerweile größter Hersteller von Mikrowellengeräten prototypisch für diesen Unternehmenstyp.

- „Vernetzte Wettbewerber“ stellen Unternehmenskonglomerate von meist kleineren Firmen dar, die unter Ausnutzung vorteilhafter Standortbedingungen zu Marktführern im Verkauf einzelner, meist niedrigtechnologischer Produkte werden. Ein Netz von Hunderten Unternehmen in Wenzhou vereint beispielsweise im Bereich des Verkaufs von Feuerzeugen einen Weltmarktanteil von $70 \%$ auf sich und verdrängte wichtige asiatische Konkurrenten.

- Bei „Technologieführern“ handelt es sich um Unternehmen, die auch in technologisch avancierten Bereichen international wettbewerbsfähig geworden sind (wie Lenovo in der Computerindustrie, Haier im Haushaltselektronikmarkt, Huawei in der Kommunikationsausrüstung und die Firma TCL in der Herstellung von Fernsehgeräten) oder es voraussichtlich werden. Sie können auf die Unterstützung der landesinternen Forschungseinrichtungen zurückgreifen (Fan 2011).

- „Ressourcenorientierte“ Unternehmen agieren im Ausland mit dem Ziel der Sicherung von Rohstoffen. Dazu gehören die Shanghai Baosteel Group, die ein großes Stahlwerk in Brasilien gebaut hat, oder die Firmen Sinopec und China National Petroleum Corporation mit Beteiligungen an Öl- und Erdgasprojekten in mehreren Ländern, darunter in geopolitisch sensiblen Staaten wie Irak oder Iran.

Da ein übergreifendes Ziel in China die Bildung nationaler Flagschiffunternehmen ist unter ,nationalen Champions“ werden protegierte Firmen verstanden, die auf der Basis starker Positionen auf dem Heimatmarkt sukzessive einen transnationalen Einfluss geltend machen -, werden die Bemühungen der Unternehmen politisch flankiert. Die Staatsführung ist dabei an einer engen Verbindung zu den neuen Unternehmensstandorten und Märkten interessiert: „An interesting reflection of this trend is the fact that virtually every overseas trip by a senior Chinese official now includes a visit to the local Huawei subsidiary“ (Williamson und Zeng 2007, S. 99). Ferner werden Subventionen und Kredite für den Einstieg in Rohstoffprojekte oder den Ankauf technologieintensiver Auslandsunternehmen gewährt. Mithilfe der staatlichen policy banks steht den chinesischen Firmen ein finanzieller Beistand zur Seite.

Die chinesischen Konzerne konzentrieren sich häufig erst einmal auf die Märkte der Schwellenländer, weshalb sie etwa in Europa noch in vergleichsweise geringem Maße wahrgenommen werden. Ziel ist es jedoch, auch die wichtigsten OECD-Ökonomien stärker zu bedienen. Einen Hintergrund für die Aktivitäten in Lateinamerika stellt der Versuch dar, Exportplattformen für einen erweiterten Zugang zum nordamerikanischen Markt aufzubauen.

Simultan befördern ressourcen- und sicherheitspolitische Maßnahmen die Aufwertung Chinas. Dies manifestiert sich in einem ausgedehnten politischen Engagement im Greater Middle East, in Asien, Afrika und Südamerika, wodurch sich die Staatsführung unter anderem eine langfristig gesicherte Versorgung mit Rohstoffen verspricht. Hieraus folgten bereits häufiger politische Konflikte besonders mit den USA, die sich im Ringen um die Sanktionen gegen den Iran im UN-Sicherheitsrat oder der Ablehnung einer UN-Intervention im Sudan widerspiegelten. Die zusammen mit Russland 
durchgeführte Blockade des UN-Sicherheitsrates hinsichtlich einer Syrien-Resolution (im Februar 2012) wird als soft balancing Chinas bewertet. Ebenso sorgte in der jüngsten Vergangenheit der Bereich der Währungspolitik für Spannungen, wie das nächste Unterkapitel aufzeigt.

\subsection{Währungsdispute}

Wie beschrieben, spielte der wirtschaftliche Einbruch in den alten Zentren der Weltwirtschaft den chinesischen Unternehmen und der chinesischen Staatsführung zu: Es wird in westlichen Volkswirtschaften aktiv um chinesische Investoren geworben und bereits stattgefundene Investitionen werden im Allgemeinen positiv evaluiert. Eine Nebenfolge der (sich verstärkenden) Going-out-Strategie wird dabei jedoch nicht immer bedacht. Sie bringt eine Verschärfung der internationalen Konkurrenz um die weltweiten Exportmärkte mit sich (Zhu und Kotz 2011). Deren Erschließung hat sich als eine zentrale Krisenlösungsstrategie großer Ökonomien erwiesen. Wirtschaftspolitisch resultierten hieraus ab 2010 aufsehenerregende Währungsrivalitäten. ${ }^{9}$

Vor dem Hintergrund einer bescheidenen Wachstumsbilanz vieler OECD-Wirtschaften auch nach 2008/2009 zielen eine Reihe entwickelter Volkswirtschaften gleichzeitig auf die Strategie des Exports ab. Um den inneren Wachstumsblockaden zu entgehen, möchte etwa die US-Regierung den Export in den nächsten Jahren beträchtlich steigern. Sie forderte auf dem G20-Gipfel in Seoul (November 2010) sogar eine (erfolglose) Begrenzung von Exportüberschüssen anderer Exportländer. Da unter der Voraussetzung einer Exportschwemme Überkapazitäten entstehen können, erhöht das die Wahrscheinlichkeit einer Verschärfung des Wettbewerbs um die Märkte. Unter anderem deshalb versuchen einige Staaten, durch eine Verbilligung der eigenen Währung Marktanteile zulasten anderer Nationen zu gewinnen. In der amerikanischen Kritik an der „unterbewerteten“ Währung des chinesischen Renminbi bzw. dem Vorwurf der Währungsmanipulation findet der ökonomische Wettbewerb einen politischen Ausdruck. Mit welchen Mitteln hierauf reagiert werden soll, bleibt zwar umstritten: Im Herbst 2011 wurde etwa von protektionistisch orientierten US-Politikern eine strittige Gesetzesvorlage eingebracht, die den schwachen Renminbi mit Ausgleichszöllen konterkarieren soll. Allerdings hat bereits die Strategie der amerikanischen Zentralbank, eine neue Phase der Quantitativen Lockerung (Quantitative Easing QE2) zu starten - und über den Kauf von Staats- und Privatanleihen die im Umlauf befindliche Geldmenge zu steigern - vergleichsweise heftige internationale Kritiken (in China und andernorts) hervorgerufen. QE2 kann den Wert des US-Dollars senken und amerikanische Exporte verbilligen.

Die chinesische Staatsführung, die, wie erwähnt, eine im internationalen Vergleich weitreichende politische Kontrolle über das chinesische Finanzsystem und den Wechselkurs ausübt, antwortet gegen die Forderung, den Renminbi aufzuwerten, mit dem Argument, dies schwäche die chinesische Konkurrenzfähigkeit, was wiederum zu sozialen

9 Die Stabilität des je eigenen Währungsraums bildet eine wichtige Komponente des globalen Wettbewerbs. Da die verantwortlichen Institutionen diese im Verhältnis zu den jeweiligen anderen Währungen durchsetzen müssen - etwa durch Strategien der Unterbewertung -, entsteht eine Währungskonkurrenz, die bis zum politischen Währungskonflikt eskalieren kann. 
Protesten mit negativen Folgen für ausländische Investoren führe. Darüber hinaus nutzt sie ihre hohen Dollar-Währungsbestände als bargaining opportunity:

While sometimes regarded as a source of potential weakness and dependency for China, dollar holdings also represent a considerable source of structural and bargaining power. This fact has enabled China to manage its relationship with the US and global governance, in line with its developmental needs, from a position of strength (Strange 2011, S. 551).

Dass diese Spannungen, etwa wenn große Volkswirtschaften erneut ökonomisch einbrechen, in ernsthafte Währungskonflikte münden, ist nicht sicher. Möglich wäre es. Wer, wie in den USA, die Vergabe von Staatsgeldern an finanzschwache Unternehmen an Lohnsenkungen und Entlassungen knüpft, droht einen „Neo-Merkantilismus“ anzuregen, der unter Bedingungen weltweit sinkender Nachfrage in eine verschärfte Beggar-thyneighbour-Politik umschlägt (Schmalz 2011). Zumal sich bereits in den Jahren vor der Krise die Hegemonialposition des amerikanischen Finanzsystems und des US-Dollars abgeschwächt hat: Die vor 2008 mit dem Begriff Bretton Woods II umschriebene Lage, die auf Leistungsbilanzdefiziten der Vereinigten Staaten einerseits und Leitungsbilanzüberschüssen in China/Ostasien andererseits beruhte und die Dominanz des US-Dollars trotz der Staats- und Auslandsverschuldung der USA garantierte, wird angezweifelt, und ein fragmentiertes Währungssystem wahrscheinlicher (Cohen 2009). Da dies nicht im Interesse der USA liegt - der globalen Vorherrschaft des US-Dollars verdankt der amerikanische Staat größere Kapazitäten als andere Nationen, Leistungsbilanzdefizite, einen gigantischen Verteidigungshaushalt sowie Krisen zu schultern -, deutet diese Entwicklung auf herannahende Auseinandersetzungen hin. Vorschlägen aus China zur Schaffung einer neuen Weltfinanzarchitektur und Androhungen des Abstoßens der Dollarbestände ${ }^{10}$ ist in den USA nicht grundlos mit Argwohn begegnet worden. ${ }^{11}$

10 Sollte die VR China ihre Dollarbestände zu schnell umschichten, würde deren Wert fallen und zugleich der Wert des chinesischen Renminbi steigen, was einen zentralen Wettbewerbsvorteil der Exportökonomie Chinas konterkariert. Die Staatsführung denkt mit ihren Vorschlägen daher vorwiegend an einen mittel- bis längerfristigen Prozess der Umschichtung, der die Erosion der aktuellen Leitwährung dennoch vorantreiben könnte. Es ist möglich, dass der Renminbi in absehbarer Zeit zu einer vollwertigen Reservewährung wird.

$11 \mathrm{Ob}$ sich mittelfristig eine konfrontativere Haltung der US-Regierung gegenüber China durchsetzt, hängt vom Ausgang inneramerikanischer Auseinandersetzungen ab: Während tief in die chinesische Wirtschaft eingebettete transnationale US-Konzerne an kooperativen Beziehungen mit China interessiert sind, stehen dem Kapitalgruppen skeptisch gegenüber, die sich stärker am amerikanischen Binnenmarkt orientieren. Zudem gibt es politische Auseinandersetzungen zwischen populistisch-protektionistischen Kräften und einem vorwiegend transnational ausgerichteten Machtblock. Erstere könnten den Versuch einer Krisenexternalisierung unternehmen (Nolan 2010, S. 86). 
3.3 Die VR China in den internationalen Organisationen und der ostasiatischen Regionalisierung

Die ökonomische und politische Aufwertung Chinas hat in den internationalen Organisationen einen Ausdruck gefunden, wie zuletzt an der neuen Institution der G20 abzulesen war. Bislang bemühte sich die Volksrepublik in den regionalen und globalen Institutionen um eine kooperative Rolle. Mit einer Politik der praktischen Anpassung zielte das Land auf eine normative wie politische Integration ab. Selbst wenn Dissonanz artikuliert wurde, mündete dies nicht in entsprechende alternative Institutionen.

Ein genauerer Blick deutet allerdings auf unterschiedlich ausgeprägte Konstellationen in verschiedenen Sektoren hin: Während China hinsichtlich der Finanzmarkt- und Bankenregulierung (Basel-Abkommen, International Accounting Standards Board, Financial Stability Board) inzwischen explizit als „Status-quo“-Macht bezeichnet wird, sind dagegen im Feld der Währungs- und Wechselkurspolitik wie erwähnt Rivalitäten zwischen den USA und China entstanden. Im Bereich des Handels kam es zu Funktionsstörungen in der Welthandelsorganisation. Konflikte zwischen dem „Norden“ und den Schwellenländern haben seit 2003 eine schwelende Krise der WTO bewirkt. Hinsichtlich der Regulierung von Wissen und geistigem Eigentum hat China internationale Standards akzeptiert (TRIPS-Abkommen), es existieren jedoch Auseinandersetzungen und Klagen westlicher Unternehmen über die mangelnde Durchsetzung dieser Standards. In den Feldern der Entwicklungspolitik haben die Ablehnung des „Washington Consensus“ sowie die Reform des IWF, insbesondere der Stimmrechtsverteilung, ein Gewicht der Volksrepublik offenbart, das in ein ergebnisoffenes Ringen um neue Entwicklungspolitiken mündet (Chorev und Babb 2009). ${ }^{12}$ Wie das spektakuläre Scheitern der Klimakonferenz in Kopenhagen Ende 2009 im Bereich der Umweltpolitik illustrierte, verfügt China mittlerweile über ausreichend Blockademacht, um relevante politische Entscheidungen zu beeinflussen. ${ }^{13}$

Vor allem in Asien trägt die VR China zur Bildung von regionalen Institutionen bei, die eine größere Unabhängigkeit vom Westen bzw. von den USA besitzen oder erreichen können. ${ }^{14}$ Außerordentlich deutlich zeigt sich dies am Aufstieg Chinas in Ostasien, was

12 Das entwicklungspolitische Modell Chinas gilt anderen Schwellenländern als Muster. Ob und inwiefern hieraus ein Übergang in eine postliberale Weltordnungsphase eines „Beijing Consensus" resultiert, ist aber alles andere als sicher.

13 Das gilt bereits länger für das UN-System. Die chinesische Außenpolitik ähnelt damit in Teilen durchaus einer „realistischen“ Praxis: Die (nicht immer offen artikulierte) Einschätzung, die internationalen Beziehungen seien wesentlich durch Machtpolitiken und zwischenstaatliche Konkurrenzen gekennzeichnet, läuft auf eine Strategie des Bilateralismus und des instrumentellen Multilateralismus hinaus. Letzterer erfolgt nach der Maßgabe, mit mehreren Länder gemeinsam zu agieren, wenn es als sinnvoll erachtet wird - zugleich aber planmäßig Vorteile aus der Mehrdeutigkeit der auf Kompromissen beruhenden internationalen Abkommen zu ziehen und sich der Beschränktheit internationaler Zusammenarbeit bewusst zu bleiben.

14 Dazu gehört die 2001 mit Russland und zentralasiatischen Ländern gegründete, sicherheitspolitisch motivierte Shanghai Cooperation Organization (SCO), die im Westen zunehmend als Äquivalent zur Stellung der NATO in Zentralasien betrachtet wird. Die SCO hat den regionalen Einfluss der USA eingeschränkt (Swanström 2010). 
nach Jahrzehnten der heftigen Anfeindungen und Gegnerschaften umso bemerkenswerter scheint. In Ostasien bilden die Association of Southeast Asian Nations (ASEAN), das ASEAN-Regionalforum sowie der Ostasiengipfel und die ASEAN-plus-3-Gespräche (mit China, Japan, Südkorea) zurzeit Angelpunkte einer wirtschaftlichen Zusammenarbeit und der Entstehung eines partiellen Sicherheitsbündnisses. Die VR China erweist sich diesbezüglich immer mehr als führende Kraft.

Die Einbindung in den ostasiatischen Raum erfordert schon allein deshalb eine politische Einflussnahme, um ökonomische Instabilitäten zu bewältigen. Die Führung Chinas visiert darüber hinaus ein makroregionales Szenario an, in dem es seine neu gewonnene ökonomische Stärke auch politisch wirksam ausspielen und (in einem gewissen Ausmaß) institutionalisieren kann. Wegweisend war der erste Ostasiengipfel Ende 2005 in Kuala Lumpur, der ohne Beteiligung der Vereinigten Staaten stattfand. Die chinesischen Unterhändler hatten dies gegen den erklärten Willen Japans durchgesetzt (Dosch 2010, S. 75).

Kündigen sich also das Ende des von den USA protegierten japanzentrierten Regionalismus und die Entstehung einer „sinozentrierten“ Region an? Im Falle der historischen Konfrontation mit Taiwan ist es bereits zu einer Entschärfung des Konflikts gekommen. Eine Art ökonomische Wiedervereinigung hat begonnen. Dabei begünstigen einander ähnelnde politisch-ökonomische Architekturen die Integration. Die politische Ökonomie Taiwans stellt ähnlich wie der nachholende Staatskapitalismus auf dem Festland ein enges Beziehungsgeflecht von Partei, Regierung, Verwaltung und unternehmerischen Aktivitäten dar. ${ }^{15}$

Insgesamt resultiert aus den wachsenden Anstrengungen Chinas in Ostasien jedoch keine unhinterfragte hegemoniale Führung. Zwar haben die geschickten außenpolitischen Initiativen eine signifikante Verschiebung des Einflusses der Großmächte in der Region zur Folge. Doch auch wenn sich die Wahrnehmung gegenüber den USA verschlechtert hat und chinesische Unternehmen oder Politiker mit einer ähnlichen Sympathie empfangen werden, wie sie einstmals nur Japanern oder Amerikanern sicher war (vgl. Kurlantzick 2008), stellen die regionalen Organisationen und häufig informellen Regimes Ostasiens selbst einen gegenläufigen Trend zu den Führungsabsichten Chinas dar. Sie bilden einen relativ inkohärenten, umkämpften Regulierungszusammenhang. ${ }^{16}$

Insbesondere das weiterhin mächtige Japan behindert die Herausbildung einer unhinterfragten Führungsrolle der Volksrepublik. China und Japan sehen in der ostasiatischen Regionalisierung ein Mittel, um den jeweils eigenen Einfluss zu vergrößern, ohne dabei dem anderen Machtpol die Perspektive einer parallel erfolgenden Machtvergrößerung in

15 Allerdings bedeutet das noch keine weitgehende politische Integration. Peking nimmt zwar gegenwärtig keine aggressive Konfrontationshaltung ein, bekräftigt jedoch weiterhin die Zugehörigkeit Taiwans zum Hoheitsgebiet Festlandchinas und strebt durch eine proaktive Ostasienpolitik die Reduzierung des taiwanesischen Einflusses in der Region an (Beeson 2009).

16 Die zwischenstaatliche Kooperation in Ostasien orientiert sich noch immer vorwiegend am Modell bilateraler Beziehungen. Das 2010 wirksam gewordene Freihandelsabkommen zwischen China und den ASEAN-Ländern (CAFTA) ist auch den Rivalitäten zwischen China und Japan geschuldet, die in der Form eines kompetitiven Regionalismus, d.h. differierenden Konzepten der regionalen Integration und Ordnungsbildung, zum Ausdruck kommen (Munakata 2006, S. 133). 
Aussicht zu stellen. ${ }^{17}$ Ebenso wirken die Politik der Vereinigten Staaten und die nach wie vor überlegene amerikanische Sicherheitsarchitektur in Asien chinesischen Ambitionen entgegen. Die Fortführung des engen US-Bündnisses mit Japan und eine Politik der bilateralen Verhandlungen mit den jeweils einzelnen Ländern stoßen nicht nur in Südkorea auf Resonanz. Auf dem ASEAN-Regionalforum in Hanoi (Juli 2010) griffen die Mitgliedsländer die geopolitischen Ansprüche Chinas im Südchinesischen Meer auch deshalb scharf an, weil sie auf fortwährende kooperative Beziehungen mit den USA setzen.

Die mehr und mehr durch China aktivierte regionale Kooperation wird also durch Konkurrenzverhältnisse sowie divergierende Interessenkoalitionen und wechselseitiges Misstrauen durchzogen. ${ }^{18}$ Selbst der Taiwanfrage ist vor diesem Hintergrund alles andere als ein geebneter Weg in eine Wiedervereinigung beschieden, wenn etwa Argumente amerikanischer Politikberater zutreffen, dass gerade die Befriedung der Taiwanfrage die Rivalität mit den USA vergrößert:

As U.S. General Douglas MacArthur put it, Taiwan is an , unsinkable aircraft carrier' midway up China's seaboard. From there [...] an outside power such as the United States can, radiate“ power along China's coastal periphery. If Taiwan returned to the bosom of mainland China, the Chinese navy not only would suddenly be in an advantageous strategic position vis-à-vis the first island chain but also would be freed up to project power beyond it to an unprecedented degree. The adjective, multipolar" is thrown around liberally to describe the next world order; only the fusing of Taiwan with the Chinese mainland would mark the real emergence of a multipolar military order in East Asia (Kaplan 2010).

Viel mehr als an ein einträchtiges Miteinander erinnert die Lage in Ostasien trotz einer fortschreitenden transnationalen Verflechtung an eine instabile Konstellation, die einen Spielraum für destruktive Konkurrenzbeziehungen und bilaterale Konflikte lässt.

\section{Fazit}

Die vorgelegte Argumentation kann weder eine erschöpfende Analyse der komplexen Integration Chinas in Weltwirtschaft und -politik noch umfassende Prognosen hinsichtlich des weiteren Aufstiegs der Volksrepublik leisten. Sie erhebt allenfalls den Anspruch, folgende Punkte untermauert zu haben:

Erstens liefert eine an politökonomischen Ansätzen orientierte Perspektive wertvolle Einsichten in charakteristische Merkmale der nachholenden Entwicklung Chinas und seine außen(-wirtschafts-)politischen Präferenzen. Im Unterschied zu herkömmlichen, machtzentrierten Perspektiven in der Disziplin der IB rücken derart sozioökonomische

17 Diese für die Region Ostasiens grundlegende Spannung hat sich im Herbst 2010 bei Zwischenfällen im Ostchinesischen Meer und in einem Konflikt um einen angeblichen Lieferstopp der für die japanische High-Tech-Industrie unentbehrlichen Seltenen Erden wieder einmal entladen.

18 Wie der fortdauernde Koreakonflikt zeigt, darf dabei der Prozess der Neutralisierung zahlreicher zwischenstaatlicher Konflikte nicht überbewertet werden. 
Sachverhalte bzw. deren Verknüpfungen mit politischen Dimensionen stärker in den Vordergrund.

Es wurde gezeigt, dass der chinesische (Staats-)Kapitalismus nicht zuletzt auf Grundlage seiner tief greifenden weltwirtschaftlichen Integration erhebliche Machtkapazitäten entwickelt hat, die die politische Führung selbstbewusst unter der Prämisse der wirtschaftlichen und geopolitischen Aufwertung einsetzt. Im Unterschied zu den historischen Erfahrungen anderer Entwicklungs- und Schwellenländer hatte die wirtschaftliche Öffnung keine einseitigen wirtschaftlichen Abhängigkeiten Chinas und Einschränkungen der politischen Handlungsspielräume zur Folge. Vielmehr ebnete dies einer Vergrößerung sowohl der Machtkapazitäten des Staates den Weg, als auch der einiger chinesischer Unternehmen, die sich zu ernsthaften Wettbewerbern etablierter Konzerne entwickeln. Im ostasiatischen Regionalisierungsprozess hat die chinesische Staatsführung bereits eine freilich nicht unhinterfragte Führungsrolle übernommen.

Zweitens verkörpert der rasante Aufstieg der Volksrepublik trotz vielfacher wechselseitiger Abhängigkeiten gegenüber den ,alten“ Zentren des Kapitalismus und kooperativer Bemühungen einen spannungsreichen Prozess. Die neue Großmacht agiert in einem instabilen, von den USA dominierten Weltsystem. Die Fragilität des Globalisierungsprozesses, die mit dem Fortbestand des Systems der Einzelstaaten und weiteren, nicht aufhebbaren Unsicherheiten einhergeht, befördert national ausgerichtete chinesische Standortpolitiken, die häufig eine außenwirtschafts- und manchmal sicherheitspolitische Flankierung erhalten und nicht immer im Einklang mit anderen nationalen Standortinteressen verlaufen. Zwar verfügt die Volksrepublik nicht über ausreichend Machtkapazitäten, um relevante Veränderungen im globalen Regulierungsgefüge durchzusetzen. Allerdings gewinnt die Machtelite in Peking an Einfluss in Ostasien, der dynamischsten Wirtschaftsregion der Erde, und besitzt in den internationalen Organisationen eine zunehmende Blockademacht, die zur Sicherung ihrer als nationale Interessenlage definierten Haltung beiträgt. Im Verhältnis zu den USA hat sich derart ein Verhältnis der wechselseitigen, aber angespannten Abhängigkeit etabliert, wie jüngst Währungsrivalitäten illustrieren. Es zeigt sich, dass wirtschaftliche Interdependenzen unter anderem aufgrund der Existenz vieler staatlich protegierter Währungsräume nicht zwangsläufig Win-win-Konstellationen hervorbringen bzw. auf Dauer gestellt werden können.

Die Staatsführung Chinas schwankt insgesamt zwischen einem eher nicht intendierten Risiko der Herausforderung und einer pragmatischen Anpassung an den Führungsanspruch der USA. Die gegenwärtig unwahrscheinliche Eskalation von Rivalitäten in zwischenstaatliche Militärkonflikte sollte daher nicht zur Vernachlässigung der vielschichtigen Konkurrenz- und Konfliktformen unterhalb der Schwelle des manifesten geopolitischen Konflikts führen, die das Verhältnis zwischen dem aufstrebenden China und dem global vorherrschenden US-amerikanischen Staat kennzeichnen.

Noch dominieren im Verhältnis zwischen China und den Vereinigten Staaten glücklicherweise die reziproken wirtschaftlichen Abhängigkeiten und die Bemühungen um ein wirtschaftliches und politisches Miteinander. Verschiedene Faktoren wie eine weitere Machtverschiebung in Ostasien, die Erosion des amerikanisch dominierten Regulierungsgeflechts auf globaler Ebene, eine Zunahme des politischen Misstrauens und des Wettbewerbs der Standorte auch jenseits handels- und währungspolitischer Konflikte, beispielsweise in Fragen der Ressourcensicherung sowie die schwer zugunsten aller Beteiligten zu 
regulierenden Krisenprozesse der Weltwirtschaft könnten dieses angespannte Miteinander allerdings unterlaufen. Destruktive Konkurrenzbeziehungen wären eine wahrscheinliche Folge.

\section{Literatur}

Alden, C. (2010). China's new engagement with Africa. In R. Roett \& G. Paz (Hrsg.), China's expansion into the western hemisphere: Implications for Latin America and the United States (S. 213-235). Washington: Brookings Institution Press.

Arrighi, G. (2008). Adam Smith in Beijing: Die Genealogie des 21. Jahrhunderts. Hamburg: VSA.

Beeson, M. (2009). Hegemonic transition in East Asia? The dynamics of Chinese and American power. Review of International Studies, 35(1), 95-112.

Bremmer, I. (2010). The end of the free market: Who wins the war between states and corporations? New York: Portfolio.

Breslin, S. (2010). China's emerging global role: Dissatisfied responsible great power. Politics, $30(1), 52-62$.

Chorev, N., \& Babb, S. (2009). The crisis of neoliberalism and the future of international institutions: A Comparison of the IMF and the WTO. Theory and Society, 38(5), 459-484.

Cohen, B. J. (2009). The future of reserve currencies. Finance \& Development, 46(3), 26-29.

Deutschmann, C. (2009). Soziologie kapitalistischer Dynamik. MPI Scholar in Residence Lectures. www.mpifg.de/aktuelles/SiR/MPIfG-Deutschmann_0902.pdf. Zugegriffen: 15. Mai 2010.

Dittmer, L., \& Yu, G. T. (Hrsg.). (2010). China, the developing world, and the new global dynamic. Boulder: Lynne Rienner.

Dosch, J. (2010). China and Southeast Asia: A new regional order in the making? In L. Dittmer \& G. T. Yu (Hrsg.), China, the developing world, and the new global dynamic (S. 61-81). Boulder: Lynne Rienner.

Fan, P. (2011). Innovation, globalization, and catch-up of latecomers: Cases of Chinese telecom firms. Environment and Planning, 43(4), 830-849.

Halper, S. (2010). The Beijing consensus: How China's authoritarian model will dominate the twenty-first century. New York: Basic Books.

Huang, X. (2007). The invisible hand: Modern studies of international relations in Japan, China, and Korea. Journal of International Relations and Development, 10(2), 168-203.

Hung, H. (2008). Rise of China and the global overaccumulation crisis. Review of International Political Economy, 15(2), 149-179.

Hürtgen, S., Lüthje, B., Pawlicki, P., \& Sproll, M. (2009). Von Silicon Valley nach Shenzhen: Globale Produktion und Arbeit in der IT-Industrie. Hamburg: VSA.

Ikenberry, J. G. (2008). The rise of China and the future of the west: Can the liberal system survive? Foreign Affairs, 87(1), 23-37.

Jessop, B. (2009). Cultural political economy: Rethinking the linkages between culture and the political economy of variegated capitalism in a world market organized in the shadow of neoliberalism. Conference paper. Ringberg Conference on Varieties of Capitalism, 17.-19. Nov. 2009.

Jiang, S. (2008). The Chinese foreign policy perspective. In R. Roett \& G. Paz (Hrsg.), China's expansion into the western hemisphere: Implications for Latin America and the United States (S. 27-43). Washington, D.C.: Brookings Institution Press.

Kaplan, R. D. (2010). The geography of Chinese power: How far can Beijing reach on land and at sea? Foreign Affairs, 89(3). www.foreignaffairs.com/articles/66205/robert-d-kaplan/thegeography-of-chinese-power. Zugegriffen: 20. Aug. 2010. 
Katzenstein, P. J., \& Shiraishi, T. (Hrsg.). (2006). Beyond Japan: The dynamics of East Asian regionalism. Ithaca: Cornell University Press.

Kurlantzick, J. (2008). China's growing influence in Southeast Asia. In R. Roett \& G. Paz (Hrsg.), China's expansion into the western hemisphere: Implications for Latin America and the United States (S. 193-212). Washington: Brookings Institution Press.

Layne, C. (2009). The waning of U.S. hegemony: Myth or reality? A review essay. International Security, 34(1), 147-172.

McNally, C. A. (Hrsg.). (2007). China's emergent political economy: Capitalism in the Dragon's lair. London: Routledge.

Mearsheimer, J. J. (2010). Der aufziehende Sturm. Blätter für deutsche und internationale Politik, $55(10), 87-100$.

Munakata, N. (2006). Has politics caught up with markets? In search of East Asian economic regionalism. In P. J. Katzenstein \& T. Shiraishi (Hrsg.), Beyond Japan: The dynamics of East Asian regionalism (S. 130-157). Ithaca: Cornell University Press.

Naughton, B. (2007). The Chinese Economy: Transitions and Growth. Cambridge: MIT Press.

Nolan, P. (2010). America and the crossroads of capitalist globalization. Challenge, 53(6), 77-92.

Schmalz, S. (2011). Die Auswirkungen der Weltwirtschaftskrise 2008 auf das internationale Staatensystem. In T. ten Brink (Hrsg.), Globale Rivalitäten: Staat und Staatensystem im globalen Kapitalismus (S. 125-144). Stuttgart: Franz Steiner.

Schmidt, D. (2012). „From the Charm to the Offensive“: Hat China eine neue Außenpolitik? Asien, $32(122), 34-56$.

Strange, G. (2011). China's post-listian rise: Beyond radical globalisation theory and the political economy of neoliberal hegemony. New Political Economy, 16(5), 539-559.

Streeck, W. (2010). E Pluribus Unum? Varieties and commonalities of capitalism. MPIfG Discussion Paper 10/12. Köln: Max-Planck-Institut für Gesellschaftsforschung.

Swanström, N. (2010). China and Greater Central Asia: Economic opportunities and security concerns. In L. Dittmer \& G. T. Yu (Hrsg.), China, the developing world, and the new global dynamic (S. 109-128). Boulder: Lynne Rienner.

ten Brink, T. (2008). Geopolitik: Geschichte und Gegenwart kapitalistischer Staatenkonkurrenz. Münster: Westfälisches Dampfboot.

ten Brink, T. (Hrsg.). (2011). Globale Rivalitäten: Staat und Staatensystem im globalen Kapitalismus. Stuttgart: Franz Steiner.

ten Brink, T. (2012a). Überlegungen zum Verhältnis von Kapitalismus und Staatenkonkurrenz. Zeitschrift für Außen- und Sicherheitspolitik, 5(1), 97-116.

ten Brink, T. (2012b). Kapitalistische Entwicklung in China. Entstehungskontexte, Verlaufsformen und Paradoxien eines eigentümlichen Modernisierungsprozesses. Habilitationsschrift, eingereicht an der Goethe-Universität Frankfurt.

Wang, H., \& Rosenau, J. N. (2009). China and global governance. Asian Perspective, 33(3), 5-39.

Williamson, P. J., \& Zeng, M. (2007). The global impact of China's emerging multinationals. In C. A. McNally (Hrsg.), China's emergent political economy: Capitalism in the dragon's lair (S. 83-101). London: Routledge.

Yeung, H. W., \& Liu, W. (2008). Globalizing China: The rise of mainland firms in the global economy. Eurasian Geography and Economics, 49(1), 57-86.

Zhang, B. (2010). Chinese foreign policy in transition: Trends and implications. Journal of Current Chinese Affairs, 39(2), 39-68.

Zheng, B. (2005). China's ,peaceful rise“ to great-power status. Foreign Affairs, 84(5), 18-24.

Zhu, A., \& Kotz, D. M. (2011). The dependence of China's economic growth on exports and investment. Review of Radical Political Economics, 43(1), 9-32. 